\title{
Nuclear Data Relevant to the Production of Radioiodine I-123, I-125 by Indirect Route and Medical Applications
}

\author{
Iman Tarik Al-Alawy* Raghad Saadoon Mohammed \\ Al-Mustansiriyah University, College of Science, Physics department, Baghdad - Iraq \\ *Email: drimantarik@yahoo.com
}

\begin{abstract}
Keywords: Medical radioisotopes, lodine production, Cross Section, Indirect reactions, Recommended values.
\end{abstract}

\begin{abstract}
The use of radioactive Iodine plays an important role in the treatment of some diseases and diagnosis of others, since they have suitable half-life. The radioactivity emitted from the dissolution of radioactive I-123 and I-125, such as the emission of Auger electrons, positrons and gamma rays reduce the spread of these diseases. Therefore, in this work we discuss the Iodine production via indirect reactions. In order to calculate the cross sections of these reactions for the mentioned target elements, we recommended the cross sections for EXFOR library using the recom.m program, which is written in the present work using Matlab-8, the data are taken from different authors. In order to supply accurate databases for different practical purposes such as proton beam energy monitoring, I-123 and I-125 radioisotopes production, we have evaluated the cross section collected from IAEA for EXFOR library measured by different authors of ( $p, x n)$, $(\mathrm{d}, \mathrm{xn})$ nuclear process. The selected data and our recommended cross sections were developed for practical applications. Since the preliminary overview of the cross section measurements up to $160 \mathrm{MeV}$ showed that there are discrepancies between the literature results.
\end{abstract}

\section{INTRODUCTION}

The radionuclide's existence is measured in their half life. Scientists had realized that nuclear reactions would provide an unlimited amount of therapeutic radioisotopes. The first major use of a radioisotope was Iodine-131 for diagnosing and treatment of thyroid disease. Isotope technology has developed to a high degree, defining which unique properties of radioisotopes are best at particular task [1]. A medical radioisotope can be classified into two major groups, diagnostic and therapeutic radionuclide, depending on the half-life and its decay mode. The decaying properties of the radioisotopes can be also classified into two groups; $\beta^{+}$-emitters, EC and gamma-emitters. Therapeutic radioisotopes are also used in disease treatment, especially cancer, where gammaemitting isotopes are attached to some kind of carrier, such as a monoclonal antibody which targets particular cancer cells. The carrier delivers the radioisotope to the cancer site, where the gamma rays destroy the cancerous cells, with minimal damage to surrounding tissue [1,2]. This research is ongoing into the use of I-123 and I-125 radioisotopes for their following importance

\section{Iodine-123}

The gamma emitting isotope Iodine-123 (with half-life 13.2h) is used as nuclear imaging tracers to evaluate the anatomic and physiologic function of the thyroid [3]. The most widely used cyclotron produced radiohalogen is probably I-123. It has gradually replaced I-131 as the isotope of choice for diagnostic radiopharmaceuticals containing radioiodine. It gives a much lower radiation dose to the patient, and the gamma ray energy of $159 \mathrm{keV}$ is ideally suited for using in a gamma camera. The gamma ray will penetrate tissue very effectively without an excessive radiation dose. For this reason, it has in many instances replaced reactor produced I-131. A large number of radiopharmaceuticals have been labelledusing I-123, and the number is increasing [4]. One of the most promising uses of I-123 is in the imaging of monoclinal antibodies to localize and visualize tumors. However, preclinical and clinical experiences with radiolabeled antibodies have not realize the expectations regarding specificity and sensitivity of tumor localization with these agents. The 
area of active research in radioiodine is an area of basic research with only a few clinical applications. Receptor specific legends have been synthesized with I-123 for (SPECT) imaging and now with I-124 for (PET) imaging [5].

\subsection{Iodine-125}

Iodine-125 radioisotope has a half-life of 59.4d used in medical research, diagnostics and treatments. One of the most common effective medical applications of Iodine-125 is its involvement in the treatment of prostate cancer. Iodine-125 is used as nuclear imaging tracer to evaluate the anatomic and physiologic function [6]. When I-125 is used therapeutically, it is encapsulated in Titanium seeds and implanted in the area of the tumor, where it remains. The low energy of the gamma spectrum in this case $0.0355 \mathrm{MeV}$ limits radiation damage to tissues far from the implanted capsule. Iodine-125, due to its suitable longer half-life and less penetrating gamma spectrum, is often preferred for laboratory tests that rely on Iodine as a tracer that is counted by gamma counter such as in radio immunoassaying [3].

\section{RESULTS AND DISCUSSION}

The experimental data in the International Atomic Energy Agency (IAEA); (EXFOR) library leaves little doubts that the hypothesis of cross section gives an excellent account of many diverse types of nuclear reactions. Figs. $(1 \rightarrow 6)$ show the excitation functions to product radio Iodine from indirect reactions with their decay scheme. These plots are analyzed using the Matlab-8 for selected energy range that is given by different authors. The results are discussed as follows:

$$
{ }_{54}^{124} X e_{70}(p, 2 n)_{55}^{123} \mathrm{Cs}_{68} \stackrel{\left(\boldsymbol{\beta}^{+}, \boldsymbol{E C}\right)}{\longrightarrow}{ }_{54}^{123} X e_{69} \stackrel{\left(\boldsymbol{\beta}^{+}, \boldsymbol{E C}\right)}{\longrightarrow}{ }_{53}^{123} I_{70} \stackrel{(\boldsymbol{E C})}{\longrightarrow}{ }_{52}^{123} \mathrm{Te}_{71}^{*} \stackrel{\boldsymbol{\gamma - \text { emission }}}{\longrightarrow}{ }_{52}^{123} \mathrm{Te}_{71} \text { Reaction }
$$

The measured data for the excitation functions of ${ }_{54}^{124} X e_{70}(p, 2 n){ }_{55}^{123} C s_{68}$ reaction reported by Kurenkov et al. (1989) [7], Tarkanyi et al. (1991) [8], Takacs et al. (2005) [9a], Takacs et al. (2005) [9b], Takacs et al. (2005) [9c], Hermanne et al. (2011) [10a] and Hermanne et al. (2011) [10b] have been plotted, interpolated, and recalculated the recommended cross section in fine steps of $0.01 \mathrm{MeV}$ via building a recom.m program, the results are shown in fig. 1. Iodine-123 used in diagnostic single photon emission tomography (SPET) for nuclear imaging tracers to evaluate the anatomic and physiologic function of the thyroid [3]. It can be produced by ${ }_{54}^{124} X e_{70}(p, 2 n){ }_{55}^{123} C s_{68}$ reaction, ${ }_{55}^{123} C s_{68}\left(4.210 \mathrm{MeV},(1 / 2)^{+}, t_{1 / 2}=5.94 \mathrm{~m}\right)$ decay ${ }^{123}{ }_{54}^{123} X e_{69}\left(2.676 \mathrm{MeV},(1 / 2)^{+}, t_{1 / 2}=\right.$ $2.08 h$ ) the undergoes electron capture (EC 26.005\%) and positron emission $\left(\beta^{+} 7.00 \%\right)$ with decay energy $1.534 \mathrm{MeV}$, as shown in fig. 2 . Then ${ }_{54}^{123} X e_{69}$ decay to ${ }_{53}^{123} I_{70}\left(1.242 \mathrm{MeV},(5 / 2)^{+}\right.$, $t_{1 / 2}=13.2 h$ ) the undergoes electron capture (EC 87\%), and positron emission $\left(\beta^{+} 13 \%\right)$ with the decay energy $1.434 \mathrm{MeV}$. Then ${ }_{53}^{123} I_{70}$ decay to ${ }_{52}^{123} \mathrm{Te}_{71}^{*}\left(0.159 \mathrm{MeV},(3 / 2)^{+}, t_{1 / 2}=0.20 \mathrm{~ns}\right)$ undergoes electron capture (EC 100\%) with the decay energy $1.083 \mathrm{MeV}$. So ${ }_{52}^{13} T e_{71}^{*}$ reach the ground state undergoes gamma emission with the decay energy $0.159 \mathrm{MeV}$. 


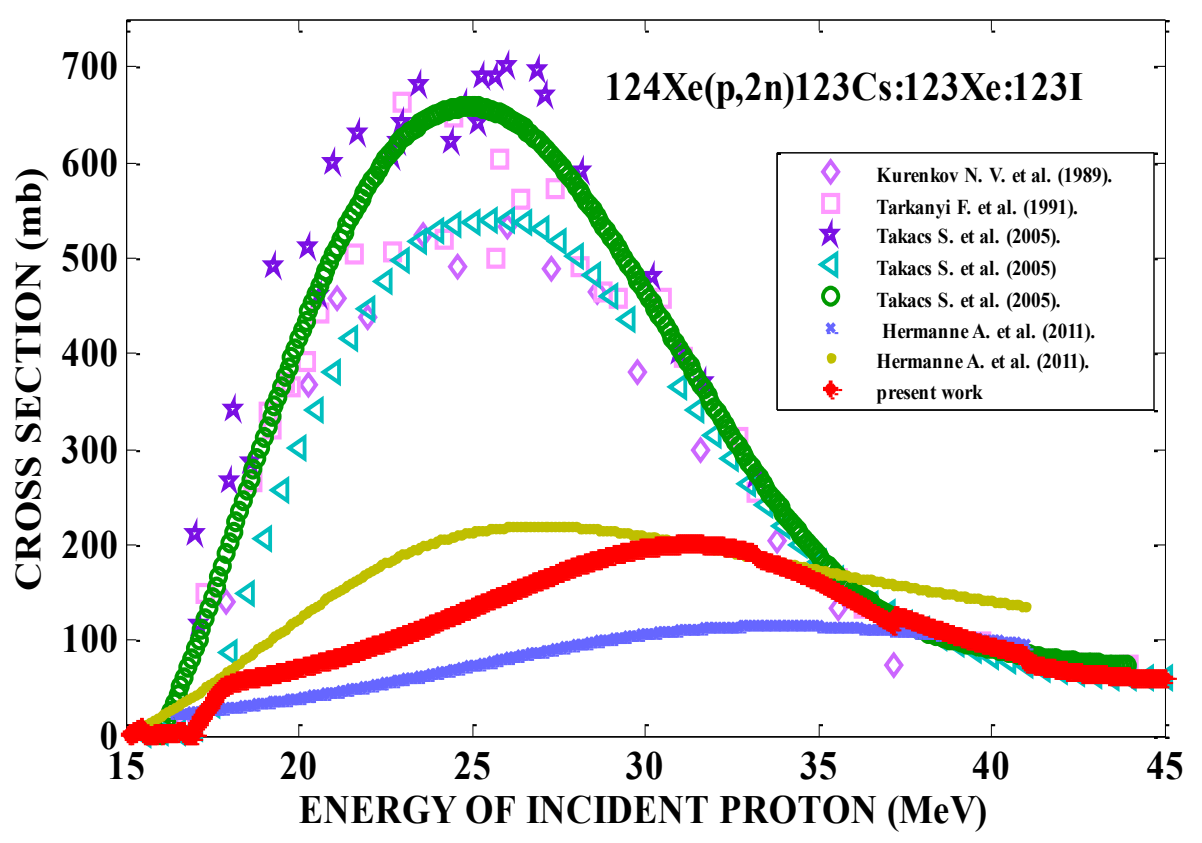

Fig. 1. The recommended cross sections of ${ }_{54}^{124} X e_{70}(p, 2 n)_{55}^{123} C s_{68}$ reaction compared with EXFOR Library.

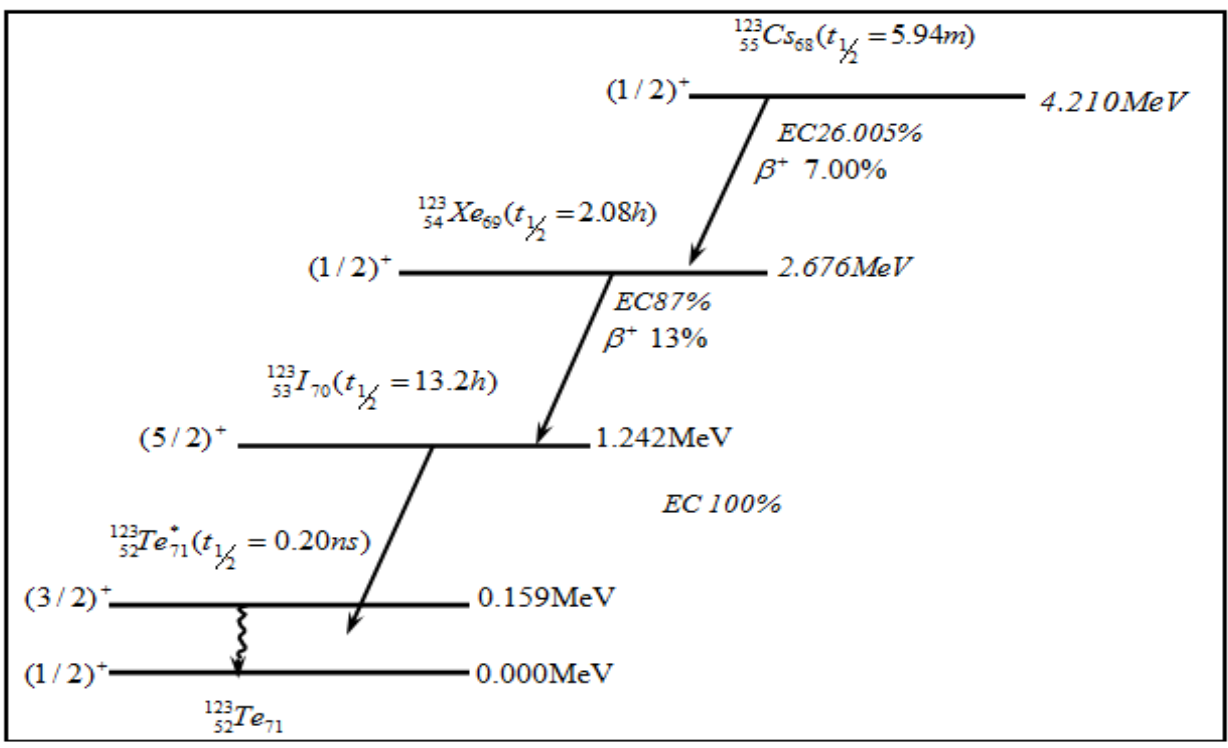

Fig. 2. Decay scheme of ${ }_{55}^{123} C s_{68}$ from ${ }_{54}^{124} X e_{70}(p, 2 n){ }_{55}^{123} C s_{68}$ reactions for the production of ${ }_{53}^{123} I_{70}$.

${ }_{53}^{127} I_{74}(p, 5 n){ }_{54}^{123} X e_{69}$ or ${ }_{53}^{127} I_{74}(d, 6 n){ }_{54}^{123} X e_{69} \stackrel{\left(\boldsymbol{\beta}^{+}, \boldsymbol{E C}\right)}{\longrightarrow}{ }_{53}^{123} I_{70} \stackrel{(\boldsymbol{E C})}{\longrightarrow}{ }_{52}^{123} \mathrm{Te}_{71}^{*} \stackrel{\boldsymbol{\gamma} \text {-emission }}{\longrightarrow}{ }_{52}^{123} T e_{71}$ Reaction

The measured data for the cross sections of ${ }_{53}^{127} I_{74}(p, 5 n)_{54}^{123} X e_{69}$ reaction reported by Wilkinsal (1975) [11], Paans et al. (1976) [12a], Diksic and Yaffe (1977) [13a], Syme et al. (1978) [14a], Syme et al. (1978) [14b], Lundqvist et al. (1979) [15a] Sakamoto et al. (1985) [16a], Lagunas Solar et al. (1986) [17a], and Deptula et al. (1990) [18a] have been plotted, interpolated, and recalculated in fine steps of $0.01 \mathrm{MeV}$ via building a recom.m program, the results are shown in fig.3-a. While the measured data for the excitation function of ${ }_{53}^{127} I_{74}(d, 6 n)_{54}^{123} X e_{69}$ reaction reported by Weinreich et al. (1974) [19], and Husson et al. (1978) [20] have been plotted, interpolated, and 
recalculated in fine steps of $0.01 \mathrm{MeV}$ by using recom.m program, as shown in fig.3-b. Therefore, Iodine-123 could be produced by ${ }_{53}^{127} I_{74}(p, 5 n){ }_{54}^{123} X e_{69}$ or ${ }_{53}^{127} I_{74}(d, 6 n){ }_{54}^{123} X e_{69}$ reaction. Hence, ${ }_{54}^{123} X e_{69}$ $\left(2.676 \mathrm{MeV},(1 / 2)^{+} t_{1 / 2}=2.08 h\right)$ decay to ${ }_{53}^{123} I_{70}\left(1.242 \mathrm{MeV},(5 / 2)^{+}, t_{1 / 2}=13.2 h\right)$ with the decay energy $1.434 \mathrm{MeV}$ the undergoes electron capture (EC 87\%), and positron emission $\left(\beta^{+} 13 \%\right)$ as shown in fig.4. Then ${ }_{53}^{123} I_{70}$ decay to ${ }_{52}^{123} \mathrm{Te}_{71}^{*}\left(0.159 \mathrm{MeV},(3 / 2)^{+}, t_{1 / 2}=0.20 \mathrm{~ns}\right)$ undergoes electron capture (EC $100 \%$ ) with the decay energy $1.083 \mathrm{MeV}$. So ${ }_{52}^{123} \mathrm{Te}_{71}^{*}$ reaches the ground state undergoes gamma emission with the decay energy $0.159 \mathrm{MeV}$.

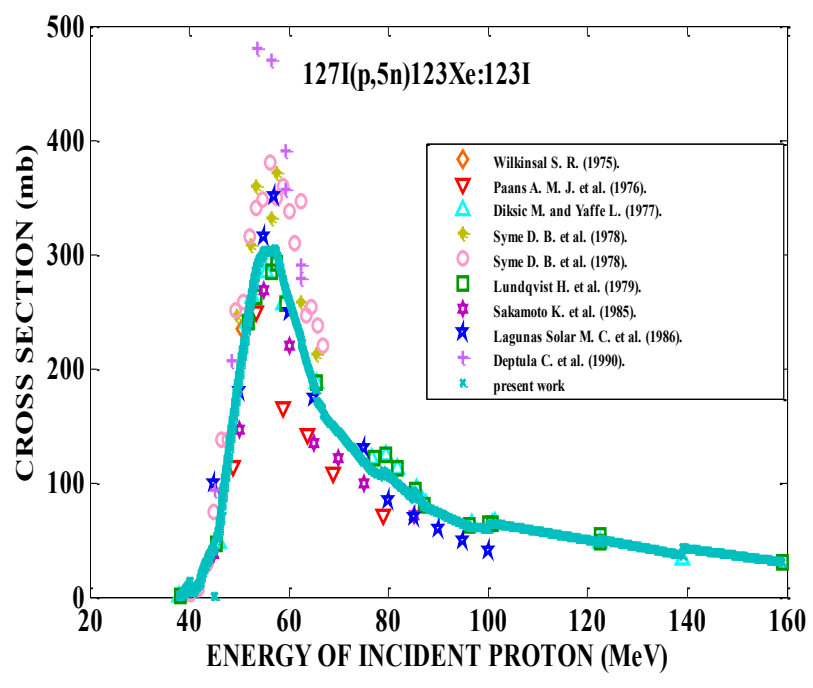

Fig. 3-a The recommended cross sections of ${ }_{53}^{127} I_{74}(p, 5 n){ }_{54}^{123} X e_{69}$ reaction compared with EXFOR Library.

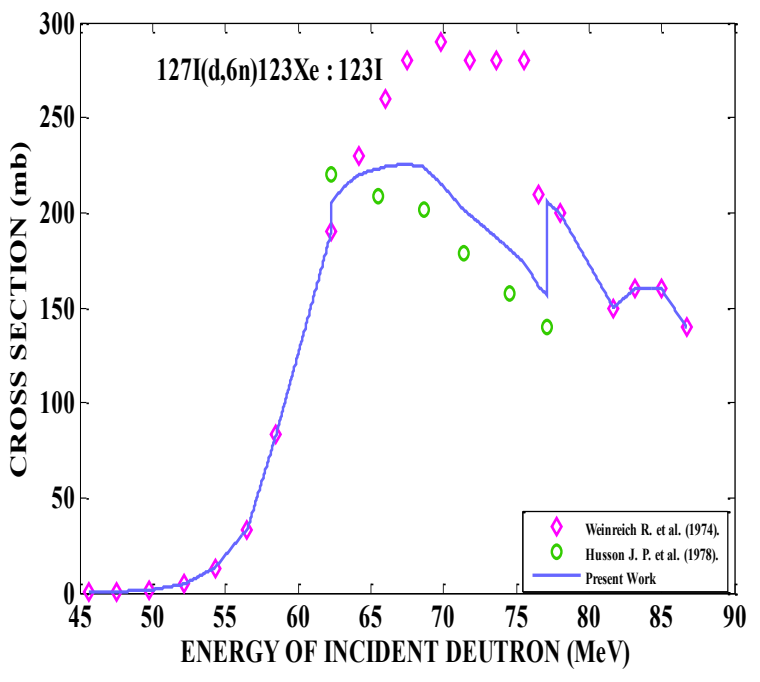

Fig.3-b The recommended cross sections of ${ }_{53}^{127} I_{74}(d, 6 n){ }_{54}^{123} X e_{69}$ reaction compared with EXFOR Library.

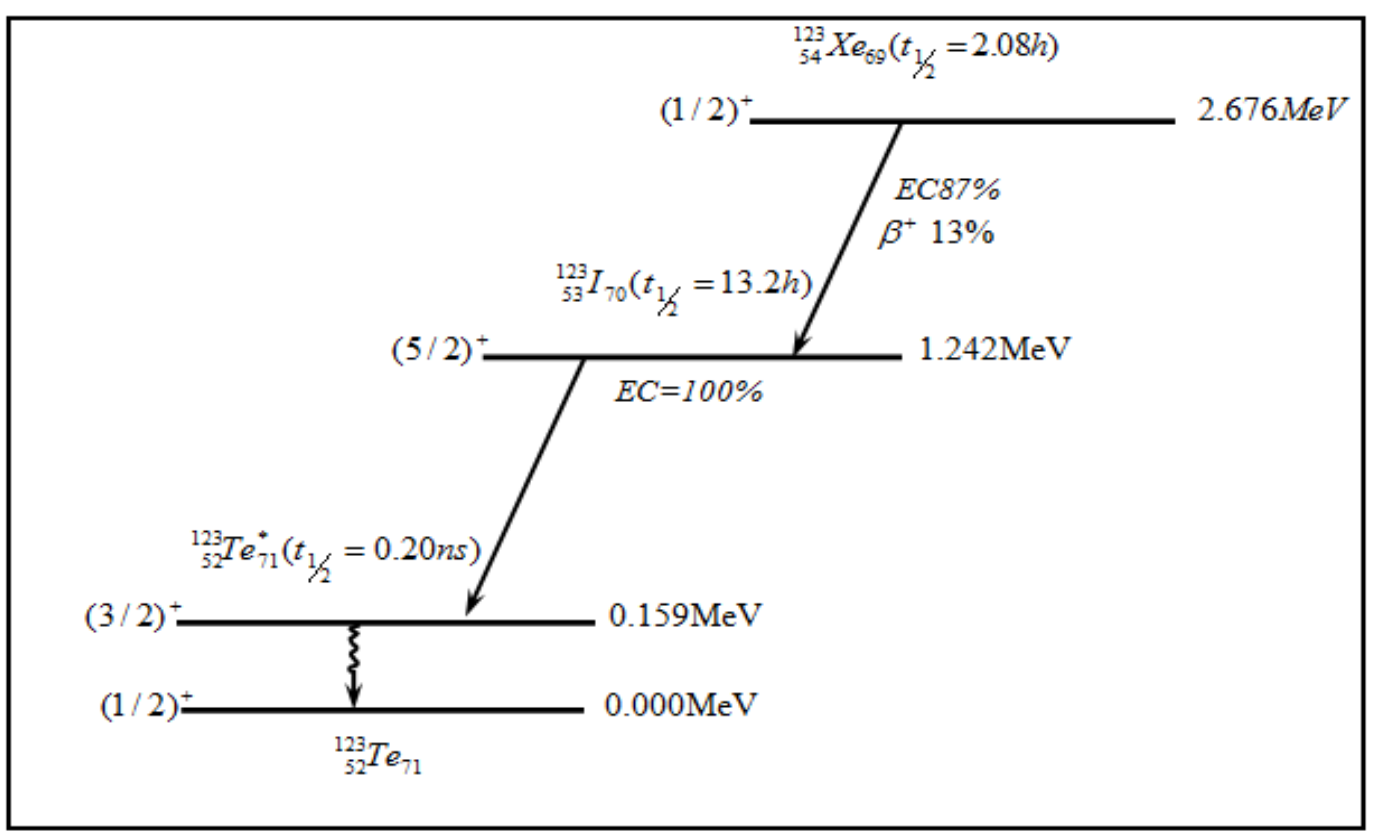

Fig. 4. Decay scheme of ${ }_{54}^{123} X e_{69}$ from ${ }_{53}^{127} I_{74}(p, 5 n){ }_{54}^{123} X e_{69}$ and ${ }_{53}^{127} I_{74}(d, 6 n)_{54}^{123} X e_{69}$ reactions for the production of ${ }_{53}^{123} I_{70}$. 


$$
{ }_{53}^{127} I_{74}(p, 3 n)_{54}^{125} X e_{71} \stackrel{\left(\boldsymbol{\beta}^{+}, \boldsymbol{E C}\right)}{\longrightarrow}{ }_{53}^{125} I_{72} \stackrel{(\boldsymbol{E C})}{\longrightarrow}{ }_{52}^{125} \mathrm{Te}_{73}^{*} \stackrel{\boldsymbol{\gamma - \text { emission }}}{\longrightarrow}{ }_{52}^{125} \mathrm{Te}_{73} \text { Reaction }
$$

The measured data for the cross sections of ${ }_{53}^{127} I_{74}(p, 3 n){ }_{54}^{125} X e_{71}$ reaction reported by Paans et al. (1976) [12b], Diksic and Yaffe (1977) [13b],Syme et al. (1978) [14c], Syme et al. (1978) [14d], Lundqvist et al. (1979) [15b], Sakamoto et al. (1985) [16b], Lagunas Solar et al. (1986) [17b], and Deptula et al. (1990) [18b] have been plotted, interpolated, and recalculated in fine steps of $0.01 \mathrm{MeV}$ via building a recom.m program, the results are shown in fig. 5. Iodine-125 used to the detection of osteoporosis and in diagnostic imaging and in tracing the impact of pharmaceutical drugs in the body and in the imaging of tumors in the planning of the receptors in the brain and in the treatment of prostate cancer, lung cancer and would like an alternative to I-131 in the treatment of brain cancer [21], so that Iodine-125 can be produced by ${ }_{53}^{127} I_{74}(p, 3 n){ }_{54}^{125} X e_{71}$ reaction. ${ }_{54}^{125} X e_{71}$ $\left(1.6530 \mathrm{MeV},(1 / 2)^{+}, t_{1 / 2}=16.9 h\right)$ decay to ${ }_{53}^{125} I_{72}\left(0.1858 \mathrm{MeV},(5 / 2)^{+}, t_{1 / 2}=59.4 d\right)$ undergoes electron capture (EC 99.7\%) and positron emission $\left(\beta^{+} 0.3 \%\right)$ with the decay energy $1.4672 \mathrm{MeV}$ as shown in fig. 6. Then ${ }_{53}^{125} I_{72}$ decay to ${ }_{52}^{125} T e_{73}^{*}\left(0.0355 \mathrm{MeV},(3 / 2)^{+}, t_{1} / 2=0.16 n s\right)$ then undergoes electron capture (EC 100\%) with the decay energy $0.1503 \mathrm{MeV}$. So ${ }_{52}^{125} T e_{73}^{*}$ reaches the ground state undergoes gamma emission with the decay energy $0.0355 \mathrm{MeV}$.

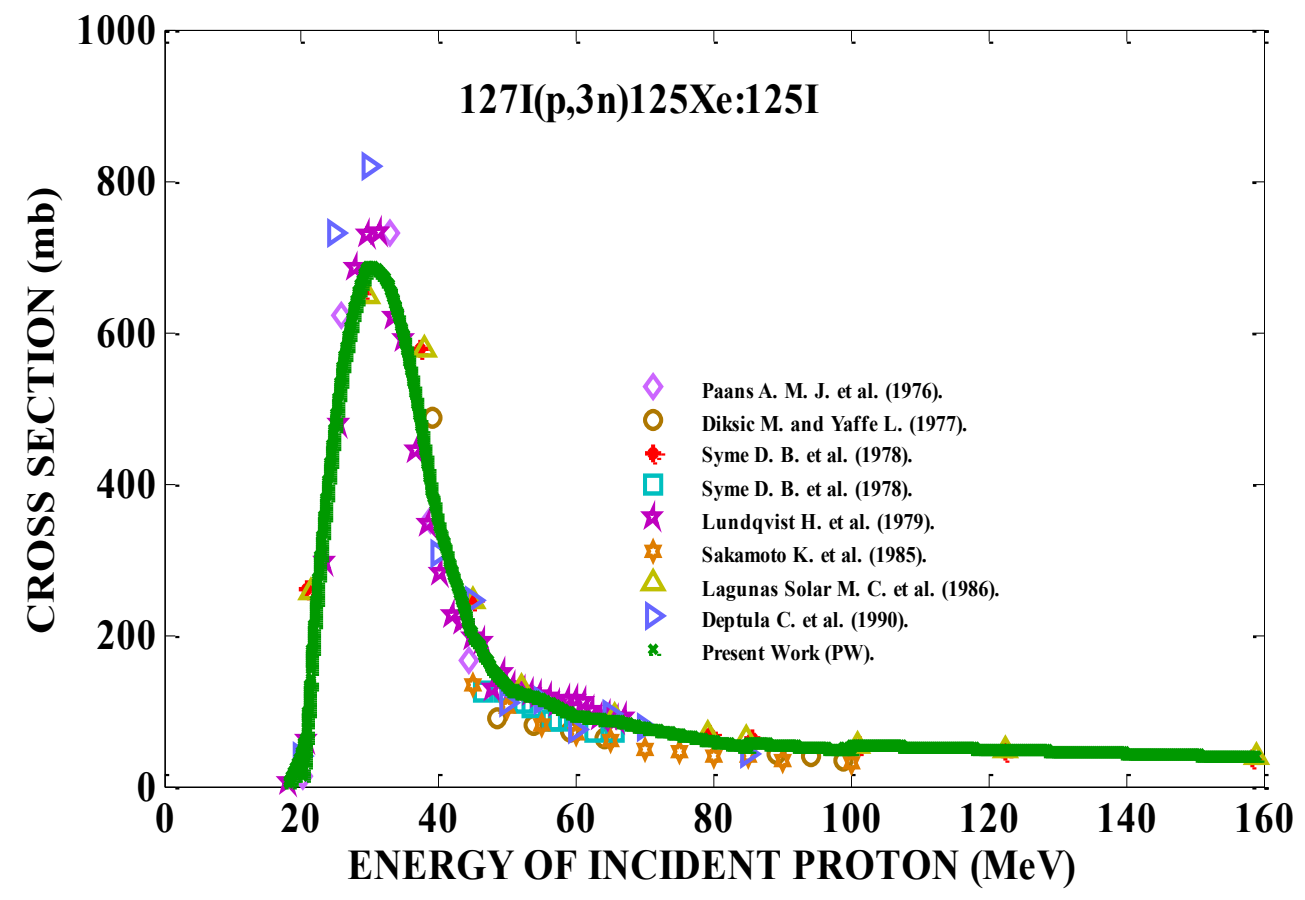

Fig. 5. The recommended cross sections of ${ }_{53}^{127} I_{74}(p, 3 n){ }_{54}^{125} X e_{71}$ reaction as compared with EXFOR Library. 


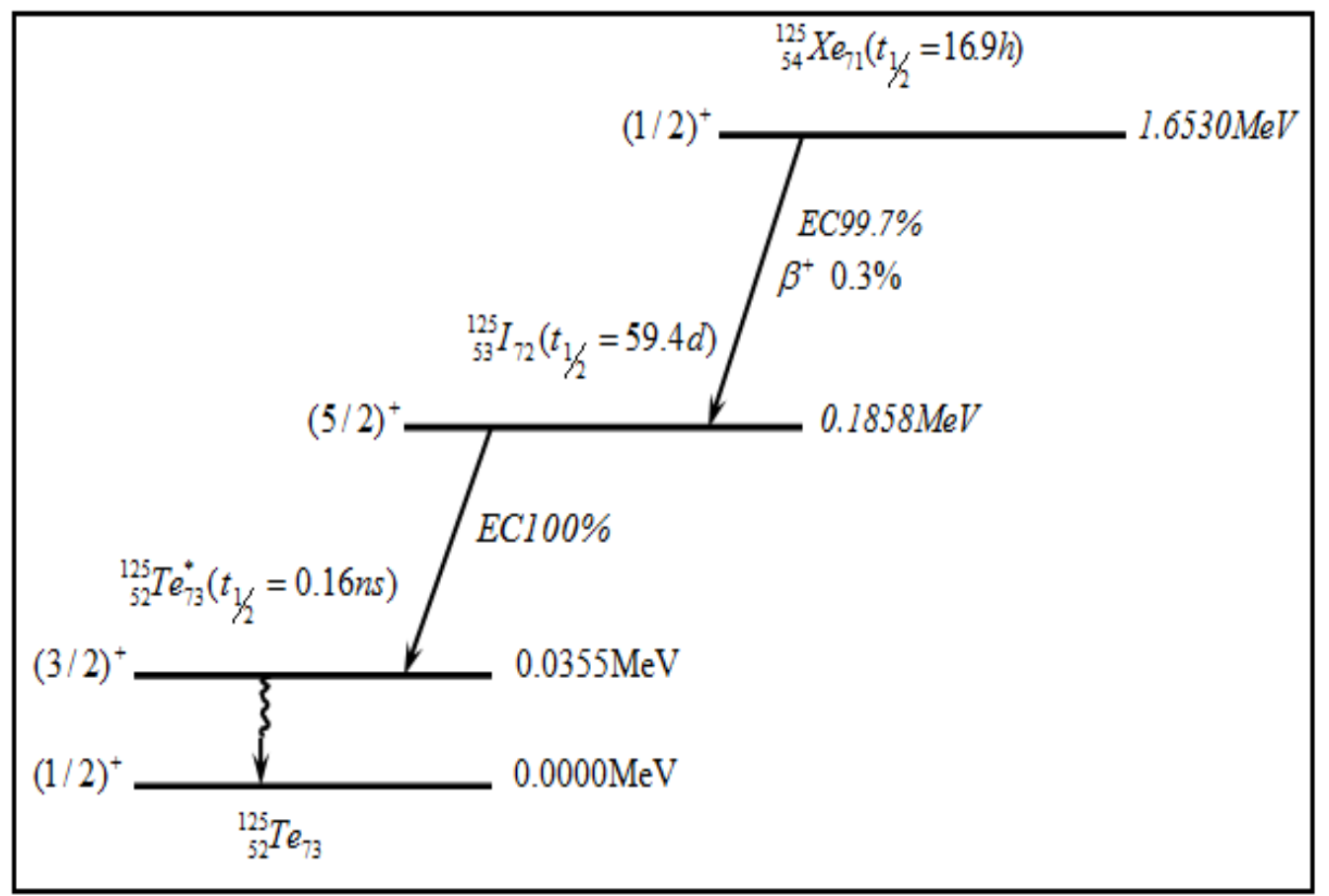

Fig. 6. Decay scheme of ${ }_{54}^{125} X e_{71}$ from ${ }_{53}^{127} I_{74}(p, 3 n){ }_{54}^{125} X e_{71}$ reaction for the production of ${ }_{53}^{125} I_{72}$.

\section{CONCLUSIONS}

Radioactive decay data play a key role in the therapeutic applications of a radionuclide I-123 and I-125 also the knowledge of the energy of the ionizing radiation is important. The effects of low energy, electrons emitted following electron capture, and positron emission decay is not negligible. Hence, for the production of I-123 from (p,2n) and I-125 form ( $p, 3 n)$ the Te-123 and Te125 undergoes gamma emission with the decay energy $0.159 \mathrm{MeV}$ and $0.0355 \mathrm{MeV}$ respectively. The characteristic of the diagnosis and treatment radioisotopes is to ensure the access of radiation to the organ for diagnostic or treated without moving to the nearby tissues by selecting a useful energy $24-28 \mathrm{MeV}$ of incident proton for the production of I-123 and I-125

\section{Acknowledgments}

The authors thank the Dean of the College of Science and the Head of Department of Physics at the Al-Mustansiriyah University for supporting this research. This work was done on the data base of an IAEA for medical radioisotope production.

\section{References}

[1] D. C. Washington, "Availability of Radioactive Isotopes: Announcement from Headquarters, Manhattan Project", Journal of Science and Technology, 103(2685), 71 (1946).

[2] (IAEA) International Atomic Energy Agency, "Charged particle cross-section database for medical radioisotope production: diagnostic radioisotopes and monitor reactions", No. 1011-4289, Vienna, 4, (2001).

[3] V. R. Narra, R. W. Howell, R. S. Harapanhalli, K. S. Sastry and D. V. Rao, "Radiotoxicity of some Iodine-123, Iodine-125, and Iodine- 131-labeled compounds in mouse testes: Implications for radiopharmaceutical design", Journal of Nuclear Medicine, 33(12), 2196 (1992). 
[4] E. Rault, "Comparison of Image Quality of Different Iodine Isotopes (I-123, I-124 and I-131)", Journal of Cancer Biotherapy and Radiopharmaceuticals, 22(3), 423-430 (2007).

[5] R. M. Lambrecht and A. P. Wolf, "Cyclotron and Short-Lived Halogen Isotopes for Radiopharmaceutical Applications. In New Developments in Radiopharmaceuticals and Labelled Compounds", (IAEA) Vienna, 1, 275-290 (1973).

[6] H. Ragde and L. Korb, "Brachytherapy for Clinically Localized Prostate Cancer", Journal of Seminars in Surgical Oncology, 18, 45-51 (2000).

[7] N. V. Kurenkov, A. B. Malinin, A. A. Sebyakin and N. I. Venikov, "Excitation functions of proton-induced nuclear reactions on Xe-124.production of I-123", Journal of Radioanalytical and Nuclear Chemistry, Letters, 135, 39 (1989).

[8] F. Tarkanyi, S. M. Qaim, G. Stocklin, M. Sajjad, R. M. Lambrect and H. Schweickert, "Excitation function of $(\mathrm{p}, 2 \mathrm{n})$ and $(\mathrm{p}, \mathrm{pn})$ reactions and differential integral yield of I-123 in proton induced nuclear reactions on highly enriched Xe-124", Journal of Applied Radiation and Isotopes, 42, 221 (1991).

[9a,b,c] S. Takacs, F. Tarkanyi and A. Hermanne, "Validation and upgrade of the recommended cross section data of charged particle reactions to produce gamma emitter radioisotopes", Journal of Nuclear Institutes and Methods in Physics Research, Sect. B., 240, 790 (2005).

[10a,b] A. Hermanne, F. Tarkanyi, S. Takacs, R. AdamRebeles, A. Ignatyuk, S. Spellerberg and R. Schweikert, "Limitation of the long-lived Te-121 contaminant in production of I-123 through the 124Xe(p,x) route", Journal of Applied Radiation and Isotopes, 69, 358 (2011).

[11] S. R. Wilkins, S. T. Shimose, H. H. Hines, J. A. Jungerman, F. Hegedus and G. L. Denardo, "Excitation functions and yields for I-123 production using the 127I(p,5n)123-Xe reaction", Journal of Applied Radiation and Isotopes, 26, 279 (1975).

[12a,b] A. M. J. Paans, W. Vaalburg, G. VanHerk and M. G. Woldring, "Excitation function for the production of I-123 via the 127-I (p,5n)123-Xe reaction", Journal of Applied Radiation and Isotopes, 27, 465 (1976).

[13a,b] M. Diksic and L. Yaffe, "A study of I-127(p,xn) and I-127(p,pxn) reactions with special emphasis on production of Xe-123", Journal of Inorganic and Nuclear Chemistry, 39, 1299 (1977).

[14a,b,c,d] D. B. Syme, E. Wood, I. M. Blair, S. Kew and M. Perry, P. Cooper, "Yield curves for cyclotron production of I-123, I-125 and I-121 by $127 \mathrm{I}(\mathrm{p}, \mathrm{xn}) \mathrm{Xe} \rightarrow($ beta decay) $\rightarrow \mathrm{I}$ reactions", Journal of Applied Radiation and Isotopes, 29(1), 29 (1978).

[15a,b] H. Lundqvist, P. Malborg, B. Langstrom and S. NaChiengmai, "Simple production of Br77 and I-123 and their use in the labeling of [77Br]BrUdR and [123I]IUdR", Journal of Applied Radiation and Isotopes, 30, 39 (1979).

[16a,b] K. Sakamoto, M. Dohniwa and K. Okada, "Excitation functions for (p,xn) and (p,pxn) reactions on natural $79+81 \mathrm{Br}, 85+87 \mathrm{Rb}, 127 \mathrm{I}$ and $133 \mathrm{Cs}$ up to Ep=52MeV", Journal of Applied Radiation and Isotopes, 36(6), 481 (1985).

[17a,b] M. C. Lagunas-Solar, O.F. Carvacho, Bo. LiLiu and Y. Jin, "Cyclotron production of highpurity I-123.A revision of exaction functions, thin-target and cumulative yields for I-127(p,xn) reactions", Journal of Applied Radiation and Isotopes, 37, 823 (1986).

[18a,b] C. Deptula, V. A. Kalkin, Kim Sen Han, O. Knotek, V. A. Konov, P. Mikecz, L. M. Popinenkova, E. Rurarz and N. G. Zaitseva, "Excitation functions and yields for medically generator Sr-82, Rb-82, Xe-123, I-123 and Bi-201, Pb-201, Tl-201 obtained with 100MeV Protons", Journal of Nukleonika, 35, 3 (1990). 
[19] R. Weinreich, O. Schult and G. Stoecklin , "Production of I-123 Via the I-127(d,6n)Xe-123 (BETA+,EC) I-123 process", Journal of Applied Radiation and Isotopes, 25, 535 (1974).

[20] P.J. Husson, Y. Legoux and C. F. Liang, "Etude Tude Des Tuxa De formation ansynchrocyclotron D'orsa y Des isotopes a via courte D'iode et DE Xenon a usage medical", Institute department Physics. Nucleaire Orsay Progress Report No.78, 27, 1 (1978).

[21] (IAEA) International Atomic Energy Agency, "Nuclear data for the production of therapeutic radionuclides", Technical report (IAEA-TECDOC-1211), Vienna, 282(2010). 